\title{
EFFECTS OF SWING EQUATION-BASED INERTIAL RESPONSE (SEBIR) CONTROL ON PENETRATION LIMITS OF NON- SYNCHRONOUS GENERATION IN THE GB POWER SYSTEM
}

\author{
Mengran Yu*, Adam Dyśko*, Andrew Roscoe*, Campbell Booth ${ }^{*}$, Richard Ierna $^{\dagger}$, Helge Urdal $^{\S}$, Jiebei Zhu ${ }^{\dagger}$ \\ *University of Strathclyde, Glasgow, UK and contact mengran.yu@,strath.ac.uk \\ ${ }^{\dagger}$ National grid Ltd., Warwick, UK ${ }^{\S}$ Urdal Power Solutions, UK
}

Keywords: non-synchronous generation (NSG), swing equation-based inertial response (SEBIR) control, penetration level limit, power system stability.

\begin{abstract}
This paper investigates the limits to penetration levels of nonsynchronous generation (NSG) in a power system and how this may be increased. Reduced system inertia, arising from high penetrations of NSG, is one of the main issues that may increase the risk of system instability in various guises. Swing equation-based inertial response (SEBIR) control, often referred to using a variety of terms, is considered to be a potential solution that can enable converter-interfaced generation to support the system during and after disturbances. However, the effects of SEBIR on system operability and its ability to increase the NSG penetration limits and improve system strength under high NSG scenarios has not been fully investigated.
\end{abstract}

The paper presents the implementation of SEBIR control within a simplified model of the future Great Britain (GB) transmission model, created using DIgSILENT PowerFactory. Using the model, the instantaneous penetration level limits of NSG in terms of both transient and steady-state stability are investigated with and without SEBIR control applied to the NSG. The capability of SEBIR in enabling additional active power output from NSG and improving system frequency response under a loss of infeed event is investigated and it is shown how SEBIR can assist in increasing NSG penetration levels, but that further work is required to understand certain phenomena that have been observed.

\section{Introduction}

With ever-increasing requirements to reduce $\mathrm{CO}_{2}$ emissions, the installation of renewable energy sources (RES) and HVDC transmission interconnectors is increasing and will contribute a large proportion of total generation capacity in the future. According to the "Future Energy Scenario" document published by National Grid (NG) in Great Britain (GB) [1] under the "Gone Green" scenario, the instantaneous generation output from RES will increase to over $50 \%$ as a proportion of total output by 2035 . Over a similar time period, the Scotland-England interconnection capacity (which represents the "weakest" link between major zones in the GB system) is anticipated to increase by $7 \mathrm{GW}$ under the "Gone
Green" scenario [1]. This interconnection will be achieved using a mix of AC or DC transmission.

Power systems were traditionally dominated by synchronous machines which adjust their rotational speed spontaneously in response to any disturbance. This initial response is dictated by the machines' inertia and acts to maintain stability of the system, giving some time for other relatively slower acting forms of response to address any imbalance in generation and demand levels in the period following the initial disturbance. In the future, the power system is expected to evolve from a relatively predictable and controllable system, to a system dominated by NSG, which will be generally less predictable, more dynamic and potentially not so easy to operate and control [2], which will clearly introduce a number of challenges.

RES and HVDC links, which are connected to the grid via power electronics generate voltage waveforms that are synchronised with the system voltage. They are often referred to as NSG or converter-interfaced generation (CIG). The primary objective is normally to maintain a constant power/current output using "conventional" converter controllers. These controllers do not normally react to any grid rotor/phase angle variations (e.g. through modulating output active power) and consequently can be viewed as not contributing to system inertia.

Due to the anticipated increase in NSG, system strength [3], which is used as a measure of the ability of a power system to remain stable during and following disturbances, will reduce significantly with increasing integration of NSG. System strength is defined by the system's aggregated inertia, the fault levels in the system and the synchronising torque available in response to disturbances. . According to [2], the overall system inertia in GB is expected to reduce by approximately $70 \%$ in $2034 / 35$ compared to $2013 / 14$ levels at low demand periods, which can lead to potentially-hazardous consequences such as high rates of change of frequency (RoCoF) following disturbances, reduced ability of frequency containment control and various system stability issues [3]. Grid codes, such as those recently drafted by ENTSO-E [4][5] and NG [6] are critical to ensuring the secure operation and evolution of power systems in future.

Various solutions have been discussed and proposed by manufacturers and researchers, e.g. reusing existing 
synchronous generators as synchronous compensators, implementing energy storage/reserve in the system to support system frequency. However, the cost of operating synchronous compensators and energy storage are relatively higher than resolving the issues using only the energy sources on the system. Therefore, SEBIR controllers are considered technically and economically desirable. SEBIR, usually referred to by others using terms such as "synthetic inertia", "virtual inertia", "emulated inertia", is used as a generic term for all types of inertial power responses provided by NSGs.

In this paper, issues and challenges associated with increasing penetration of NSG will be investigated based using analyses of frequency responses in the GB power system to events; this is covered in section 2. Section 3 discusses the principles and implementation of SEBIR control, section 4 introduces the reduced GB transmission model. In section 5, the effects of SEBIR control and its impact on NSG penetration level limits with and without SEBIR control will be investigated and discussed. Conclusions and future work are presented in section 6 .

\section{Frequency response and future challenges}

In an electrical power system, the frequency is an important indicator of system performance and its behaviour is directly governed by the prevailing balance between active power supply and demand. Under normal conditions, where power delivered by the generation units matches the demand, system frequency is controlled at around its nominal value within specific limits, e.g. $\pm 1 \%$ of $50 \mathrm{~Hz}$ in the GB transmission system, as stated in [7]. Frequency response can be generally classified into four categories depending on the response time delay after the initiating event [8]: inertial frequency response, primary frequency response, secondary frequency response and tertiary response, as illustrated in Figure 1.

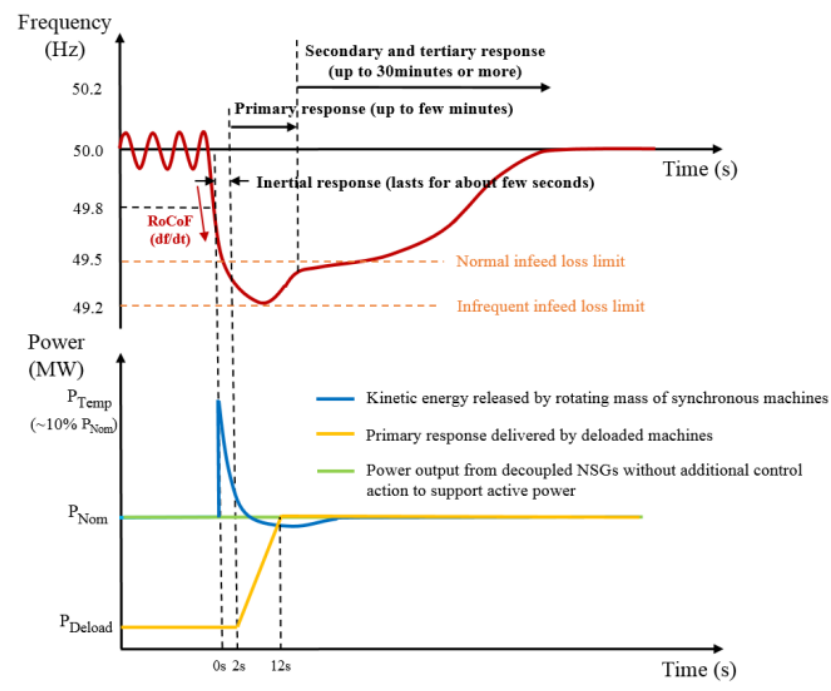

Figure 1. Typical system frequency response and corresponding power injection under a loss of infeed event [9]
As shown in Figure 1, in response to a significant loss of infeed or significant and sudden increase in loading, the system frequency will fall significantly, with an initial rate, i.e. RoCoF, which is directly related to the amount of kinetic energy stored in the rotating mass of synchronous machines in the system. Following the event, additional power is provided instantly by SMs in the system, but the amount of power will decay rapidly and dramatically and will only last for a rather short period of time, e.g. within $200 \mathrm{~ms}$ as shown in Figure 1. When the system frequency falls by more than $0.2 \mathrm{~Hz}$, generation units are contracted to provide fast-acting frequency response, i.e. primary response or governor response, normally by boosting their output, e.g. increasing the output power of deloaded SMs. According to [9], primary response will act within $10 \mathrm{~s}$ and sustain for a further time period to correct frequency deviation. Following the primary frequency response, further control actions will be executed to recover the system frequency to its nominal value, i.e. through secondary and tertiary response. This may involve starting up other power plants, importing more power over HVDC links, carrying out some load control if possible, etc.

For a robust power system, it is important that the majority of generation (and loads if possible) in the system are capable of contributing to total system inertia. However, as outlined previously, NSG sources normally do not provide an inertial response since they are decoupled from the AC grid via power electronic devices. Consequently, levels of RoCoF may increase significantly in future and the stability of the system could be far more vulnerable than at present. Furthermore, due to increases in capacities of generation units, the infrequent infeed loss limit for the GB system has been increased from its current level of $1320 \mathrm{MW}$ to 1800 MW [10], which obviously means that the overall system must be relatively stronger to cope with a larger maximum infeed loss.

\section{SEBIR control}

There have been several publications and debates relating to solutions to future reductions in system strength and how these may be addressed, where various types and implementations of SEBIR control have been proposed to enable NSG to support system frequency recovery in response to disturbances. [11] introduces control techniques to extract stored kinetic energy from the rotating elements of wind turbine generators (WTG), and this is often termed synthetic inertia. Similar concept for WTG can also be found in many documents, examples include [12][13]. [14]discuss inertia emulation control techniques for HVDC links to support system frequency by manipulating energy stored in the DC capacitors. Other terms used in the literature include artificial inertia, simulated inertia, etc. However, the principle of those control techniques are common, and are invariably based on the Swing Equation of a SM, as shown in (1),

$$
\Delta P=P_{\mathrm{m}}-P_{e}=\frac{2 H}{f_{\text {nom }}} \frac{d f}{d t}
$$


where $\Delta \mathrm{P}$ is the amount of power imbalance in the system, $P_{m}$ and $P_{e}$ are the mechanical and electrical power of the SM respectively, $H$ is the inertia constant of a synchronous generator, $f_{\text {nom }}$ is the nominal frequency of the system and $d f / d t$ is the RoCoF resulting from the power imbalance. When there is a power imbalance in the system, e.g. for a loss of infeed event, the system frequency will drop and the RoCoF will be negative; as a result, the NSG that are equipped with SEBIR control will generate the corresponding $\Delta \mathrm{P}$ by detecting the RoCoF to support the system power balance.

Note that in addition to SEBIR techniques investigated in this paper, there have been virtual synchronous machine (VSM) control techniques proposed by other authors which are purported to enable converters to behave in an almost identical fashion to actual SMs. Techniques reported include VISMA [15], Synchonverter [16], and Virtual SG [17]. These techniques offer various network stability benefits, but are not within the scope of this particular paper. It is the intention to examine these VSM techniques later in the project. In this paper, the analysis is constrained to the use of the SEBIR technique, implemented by augmenting conventional PLLsynchronised (rotating reference frame) grid-connected dqaxis-frame controllers.

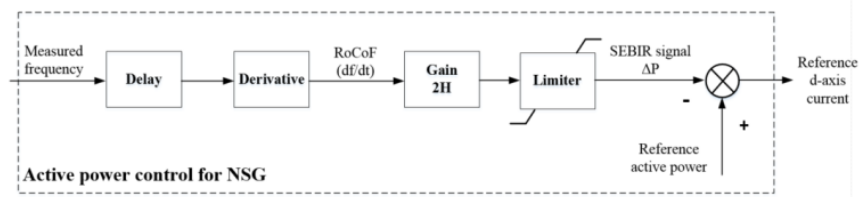

Figure 2. SEBIR control implementation in the reduced GB transmission model

Based on equation (1)Error! Reference source not found., a generic and reconfigurable SEBIR controller has been built and implemented within active power control system for static generators in the reduced GB transmission model using DIgSILENT PowerFactory, as shown in Error! Reference source not found. According to [4], NSG should operate in either frequency sensitive mode (FSM) or limited frequency sensitive mode (LFSM) to support system stability. In this model, all static generators operate in LFSM, where an active power-frequency droop will start to act when measured frequency at the generation bus exceeds $50.4 \mathrm{~Hz}$. One very important aspect of any "synthetic" technique is the delay in detecting the need for and instructing the response, which is variable and can never be truly instantaneous. The time delays associated with measuring and processing must be considered. Accordingly, a ramp limiter has been applied to vary the response speed of the SEBIR controller to reflect and investigate delays due to different controllers and energy sources "behind" the converters. A variable limit can also be set for the magnitude of the increased active power support from the SEBIR controller to reflect and investigate the impacts of different levels of "reserve capacity" which may be available from the energy sources. The SEBIR signal $\Delta \mathrm{P}$ is then added to the reference active power output. Note that the NSG models in the reduced GB transmission model are represented by static generators with vector current controllers. An example graph of output power from NSG controlled by SEBIR control is shown in Figure 3, with consideration of factors discussed above. Note that the ramps shown in the graph are an approximated trend which may vary in corresponding with different types of NSG.

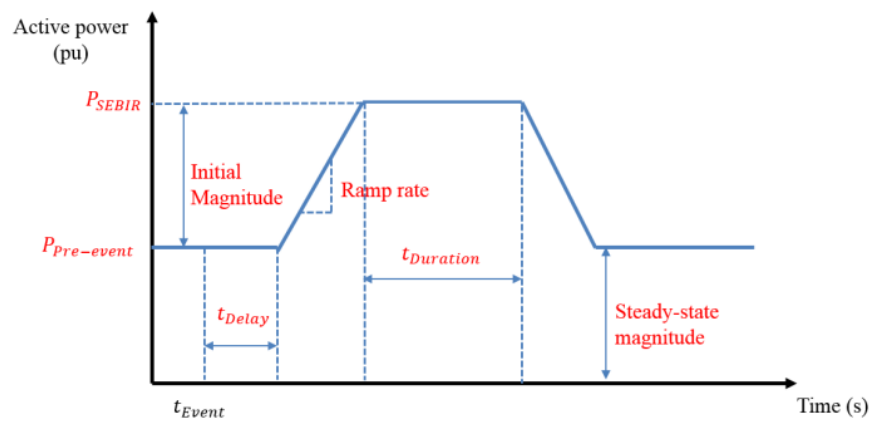

Figure 3. Example active power output from NSG equipped with SEBIR control

\section{Simulation scenarios}

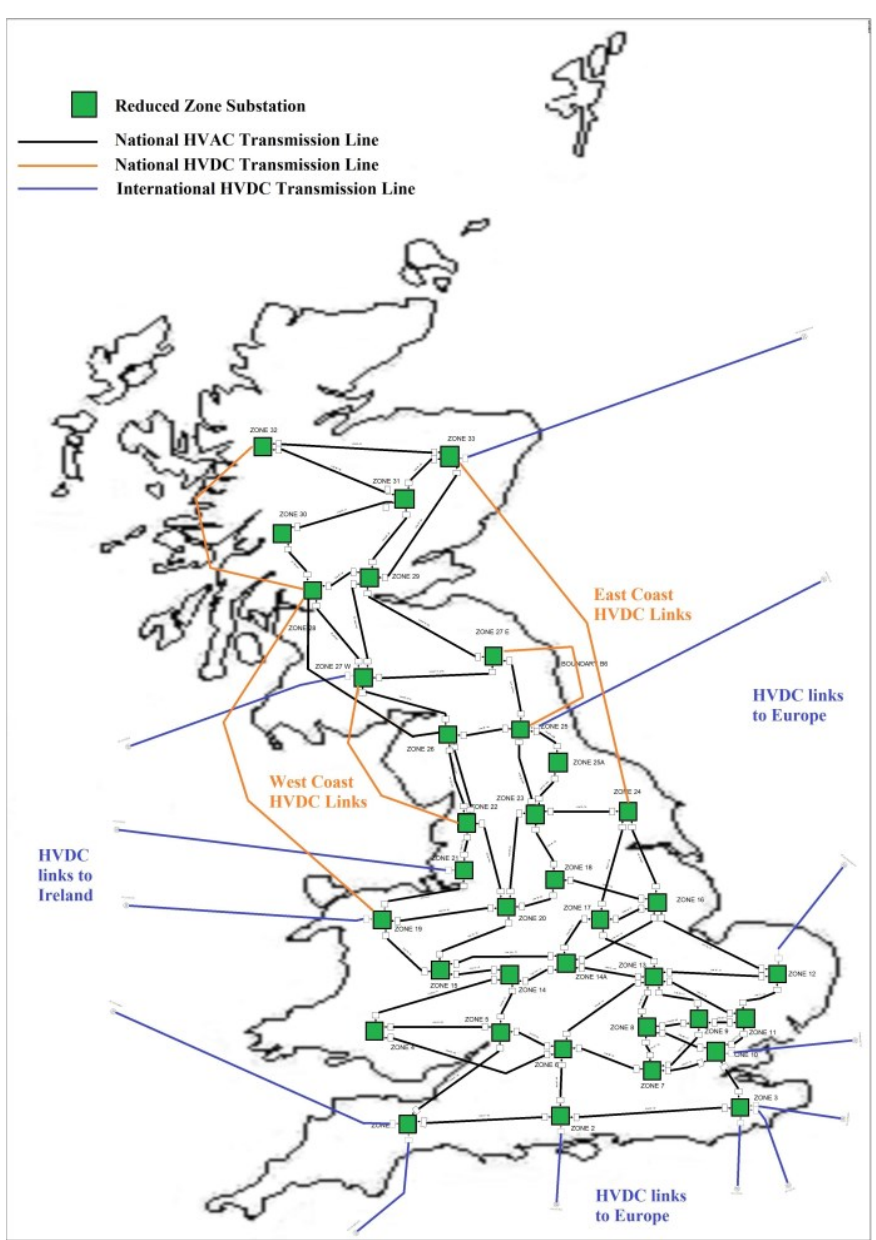

Figure 4. Reduced GB 36-bus/substation transmission model under 2030 Gone Green Scenario

The studies in this paper are based on a 36-bus equivalent network representing the National Electricity Transmission System of Great Britain, which is modelled in DIgSILENT 
PowerFactory and has been prepared by NG, as shown in Figure 4. The model is dispatched according to the GB 2030 Gone Green scenario[1]. Each node in the model represents a part of the system and is composed of relevant generation (represented as a mix of different energy sources), demand and HVDC interconnectors. Generators within each zone are categorized according to fuel types and represented by synchronous generators or static generators, including corresponding dynamic controllers as well as SVCs/STATCOMs to reflect system reinforcements under high NSG scenario in 2030. With wind farms concentrated in Scotland and offshore, and with new nuclear stations in the south of the GB power network in the future, the network will face major stability challenges arising from a distinct lack of synchronous generation located in the north of GB [2].

In this paper, scenario cases have been selected and designed to investigate response to worst-case events in the GB power network with a high penetration of NSG, e.g. at summer minimum demand under the Gone Green scenario. According to [1], the level of summer minimum demand will fall to approximately $18.5 \mathrm{GW}$. Therefore, two levels are selected to explore the NSG\% limits: $18 \mathrm{GW}$ and $26 \mathrm{GW}$. Note that the demand stated represents a gross demand which sums up loads in transmission system and embedded generation in distributed system.

Using the model, the proportion of power provided from SG and NSG can be varied to achieve different instantaneous penetration levels (IPLs) of NSG and therefore to find the limits.

\section{Results and discussion}

\subsection{System performance with SEBIR control}

In order to investigate the effects of SEBIR control on the system performance, a $1724 \mathrm{MW}$ synchronous generator located in the central element of the GB network is tripped at $2 \mathrm{~s}$. The particular generator is chosen to be as close a reflection as possible of the infrequent infeed loss, i.e. $1800 \mathrm{MW}$ in the GB power system [10]. Generation dispatch for this particular case study is: 0 import/ 0 export to/from HVDC links and $30 \%$ penetration of NSG, at a gross demand level of $26 \mathrm{GW}$. It is assumed that there is no time delay in the response of the SEBIR control (although in practice there would be some - this is being investigated in on-going and future work) and that the NSG sources are able to contribute the additional active power instructed by SEBIR control (which again may not always be possible and is being investigated in future work). The limiter of SEBIR control is set to $\pm 10 \%$ of NSG rated capacity. A base case is set up with no SEBIR control and inertia constant of the synchronous generators in the model are set to $5 \mathrm{~s}$. The inertia constant of synchronous generators and NSG is then varied to compare the relative effects. Frequency and active power output measured at the location of a wind farm in Scotland are shown in Figure 5. Note that the SEBIR control is applied to all NSG, i.e. static generators, in the model with the same settings.

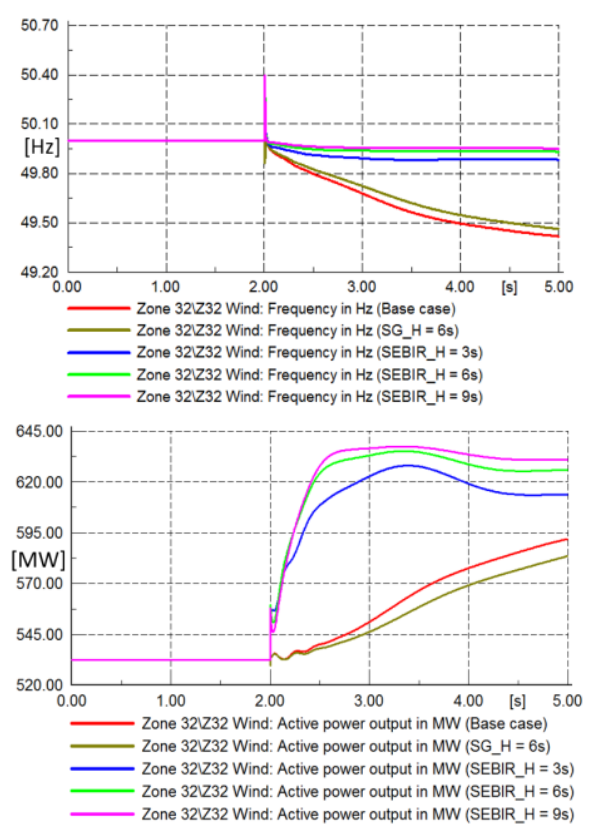

Figure 5. Frequency and active power output for a Scottish wind farm with different value of inertia constant of SEBIR control for a 1724MW loss of infeed in GB system

In the base case with no SEBIR control, the frequency falls rapidly after the disturbance. By increasing the inertia constant of all synchronous generators to $6 \mathrm{~s}$ from $5 \mathrm{~s}$, the drop is alleviated slightly as expected. Similar effects can be seen in active power output from the same wind farm. With higher inertia constants in the SEBIR control, as shown in Figure 5, the frequency drop becomes increasingly alleviated, while the magnitude of active power output from wind farm is increased with higher inertia constant in the SEBIR control. It is obvious that the SEBIR control is capable of enabling additional active power support from NSG to the system and as a result, improving system stability/strength in response to disturbances. In reality, the inertia constant that can be achieved in practice using SEBIR is limited by the amount of available stored or "extra" energy in generation source and its capability to ramp up its output. This can all be carried in the generic model that has been produced in this research and these aspects will be investigated in on-going and future work and reported at the conference.

It should be mentioned that the frequency spikes occur at the instant of the event may trigger the FSM or LFSM, which contribute a certain degree of primary response. Different sources may respond more quickly - for example HVDC links may be able to increase outputs more rapidly - this will be investigated in future work. In reality, the SEBIR measuring and processing procedures will act to make the response even slower. However, even with a slower response, the study shows the potential of this relatively simple control technique to improve system frequency stability. The effects 
of additional delays may be negative and will be studied in detail in future.

\subsection{IPL limits of NSG}

Studies in [18] have shown that the maximum NSG instantaneous penetration level (IPL) in terms of first swing stability, i.e. angular stability, is in the region of $65 \%$ of dispatched generation (MW), or $75 \%$ in terms of connected generation capacity (MVA) for the GB power system. These results were produced using the same reduced GB transmission model as used in this study. Paper [18] defines IPL as shown in equation (2), which considers the import and export through HVDC links, where $P$ represents real power generated, consumed, imported, or exported. In this paper, it is assumed that there is no import and export power via HVDC interconnectors. Although SEBIR control has been shown that it can enable NSG to contribute active power support during and after disturbances, the question of whether it is able to increase the penetration level limit of NSG remains open to debate.

$$
I P L \%=\frac{P_{N S G}+P_{H V D C_{\text {_import }}}}{P_{\text {Demand }}+P_{H V D C_{-} \text {export }}}
$$

In order to test the ability of SEBIR control to improve penetration level of NSG, a three-phase solid fault on two of the four HVAC links, i.e. a double circuit trip, between Scotland and England has been chosen to test system stability, which is commonly considered as the most severe fault that can happen in the GB network. The fault is applied at $1 \mathrm{~s}$ with a duration of $100 \mathrm{~ms}$. An integration step of $0.5 \mathrm{~ms}$ is applied in the simulation. As introduced before, three factors are selected to be investigated to explore the IPL limits of NSG in GB power system:

a) demand levels - $18 \mathrm{GW}$ and $26 \mathrm{GW}$;

b) inertia constant of the SEBIR controller - 0s, 3s, 6s and $9 \mathrm{~s}$; as well as $9 \mathrm{~s}$ of inertia constant of the synchronous generators (compared to $5 \mathrm{~s}$ in the base case).

A case study with relatively low IPL of NSG is shown in Figure 6. During the fault and after fault clearance, the system settles down relatively quickly. However, with increasing IPL of NSG, for the same event, transient instability and even steady-state instability occur, as shown in Figure 7 and Figure 8 respectively. For the transient instability case, the system is able to operate normally before the fault but becomes unstable after the fault, which indicates a loss of synchronism in the system. When the level of IPL is increased further, the system becomes unstable even before the fault event, as shown in Figure 8. It is noticeable that the waveforms immediately after the loss of stability contain high frequency components which are different from typical transient instability cases with conventional synchronous generators. The authors suspect this could be caused by certain high frequency oscillatory modes introduced by fast acting inverter controllers of NSG. Further investigation is required to verify the exact nature of those oscillations using state space linear analysis as well as careful consideration of the integration step to rule out the possibility of numerical instability of the simulation. This issue has also been reported in [18].

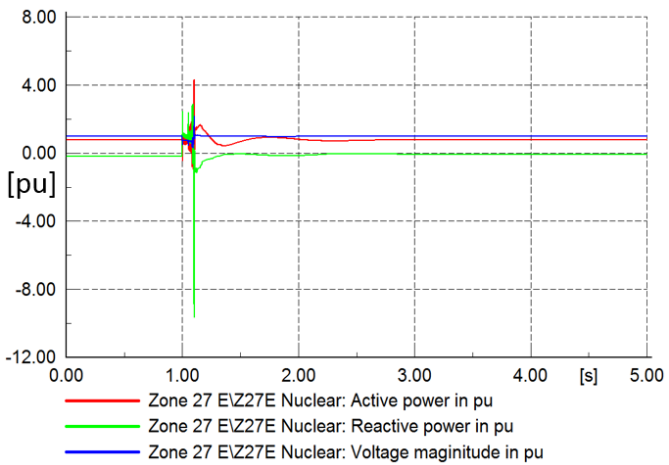

Figure 6. Response of a synchronous generator in Southeast of Scotland for a marginally stable case (Base case, $70 \%$ IPL, at 26GW demand level)

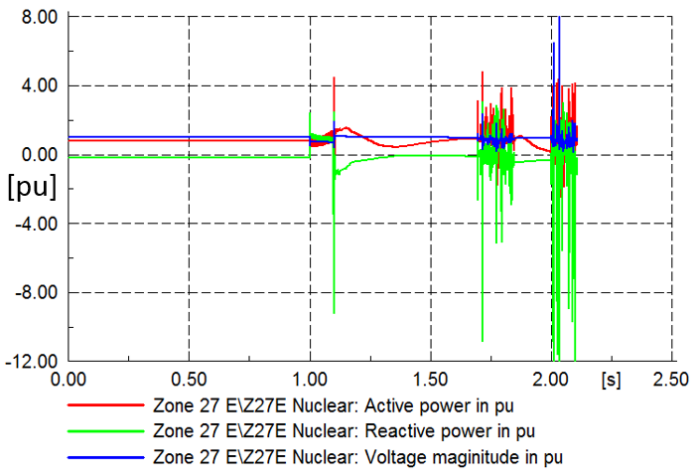

Figure 7. Response of a synchronous generator in Southwest of Scotland for an marginally unstable case in terms of transient stability (Base case, $71 \%$ IPL, at 26GW demand level)

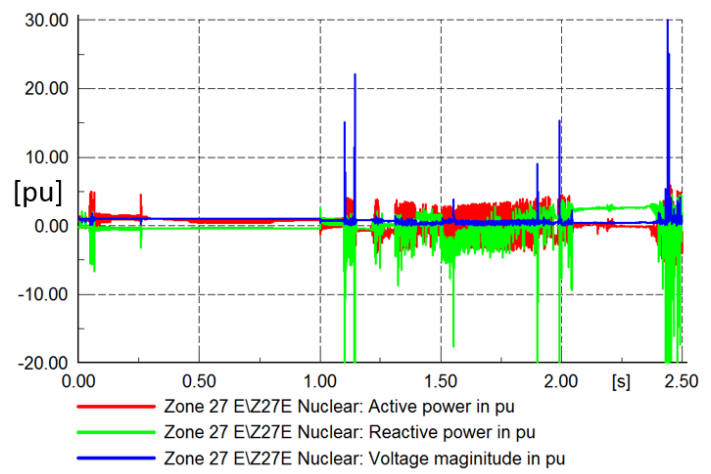

Figure 8. Response of a synchronous generator in Southwest of Scotland for an marginally unstable case in terms of steady-state stability (Base case, $88 \%$ IPL, at 26GW demand level)

Summarised results are shown in Table 1 in terms of transient stability and steady-state stability limits respectively. It can be observed that the IPL transient stability limits are improved both with increasing true inertia of the existing synchronous generators as well as SEBIR control inertia constant. Regarding steady-state stability, the IPL limits of NSG improve neither with increasing true inertia nor with SEBIR inertia in the system. The results, therefore, suggest that both 
conventional inertia and SEBIR control provide similar type of network support. This confirms certain theoretical expectation from inertial response since system inertia physically represents the stored kinetic energy in the rotating mass of synchronous machines which acts spontaneously to support system by providing instantaneous source of stabilising power during system frequency changes, and therefore, contributes to transient stability directly. With increased system inertia, the system strength and stability limits are thus expected to be higher. However, for steadystate stability which is does not involve rapid frequency changes inertial response is naturally negligible as stored kinetic energy cannot be released. In this case the limit is determined primarily by the system operating conditions, transmission system strength, line transfer capacities, or types of generator excitation controls [19]. The results fully confirm these theoretical considerations.

It is interesting to observe that at high inertia constant of SEBIR control (Case 5), the IPL limit appears to be marginally higher than the case with the same inertia constant of synchronous generators (Case 2). This can be explained by the fact that in Case 5 the SEBIR provided inertia cooperates with the inertia present in the synchronous generation, while in Case 2 inertial response is only provided by the minority synchronous generation. Nevertheless, more accurate and systematic quantification of the amount of transient stability support (especially from SEBIR and other synthetic methods not included in this paper) is needed and will be considered in the next stages of this research.

\begin{tabular}{c|c|c|c|c|c}
\hline \multirow{2}{*}{ IPL limits of NSG } & \multicolumn{2}{c|}{ Transient stability } & \multicolumn{2}{c}{ Steady-state stability } \\
\cline { 3 - 6 } & \multicolumn{2}{|c}{ Load GW } & \multicolumn{2}{c}{ Load GW } \\
\cline { 3 - 6 } & $\mathbf{2 6}$ & $\mathbf{1 8}$ & $\mathbf{2 6}$ & $\mathbf{1 8}$ \\
\hline Case 1 & $\begin{array}{c}\text { NSG\% limit without } \\
\text { SEBIR }\end{array}$ & $70 \%$ & $70 \%$ & $87 \%$ & $84 \%$ \\
\hline Case 2 & $\begin{array}{c}\text { NSG\% limit with SG } \\
\text { inertia constant =9s }\end{array}$ & $71 \%$ & $72 \%$ & $87 \%$ & $84 \%$ \\
\hline Case 3 & $\begin{array}{c}\text { NSG\% limit with SEBIR } \\
\text { control (H=3s) }\end{array}$ & $70 \%$ & $71 \%$ & $87 \%$ & $84 \%$ \\
\hline Case 4 & $\begin{array}{c}\text { NSG\% limit with SEBIR } \\
\text { control (H=6s) }\end{array}$ & $71 \%$ & $72 \%$ & $87 \%$ & $84 \%$ \\
\hline Case 5 & $\begin{array}{c}\text { NSG\% limit with SEBIR } \\
\text { control (H=9s) }\end{array}$ & $72 \%$ & $73 \%$ & $87 \%$ & $84 \%$ \\
\hline
\end{tabular}

Table 1. IPL limits of NSG in terms of transient stability and steady-state stability

\section{Conclusions and future work}

In this paper, SEBIR control has been implemented in a reduced GB transmission model using DIgSILENT PowerFactory and it has been proven that the SEBIR control is capable of enabling additional active power output from NSG and can improve system frequency response under loss of major infeed event, even though the response time of SEBIR is generally slower than inertial response from conventional SMs. The studies showed that the IPL limits in terms of transient stability are more affected by implemented of SEBIR control, while it does not affect significantly limits in terms of steady-state stability, which confirms theoretical considerations.

Further work is clearly required to understand the reasons behind the observed instability phenomena. The IPL limits will be tested under more simulation scenarios by varying settings in the reduced GB system, e.g. import and export power through HVDC links, settings of SEBIR control, applying different types of SEBIR control, etc. The amount of transient stability support provided by SEBIR control as well as other types of synthetic methods will be systematically investigated and compared.

\section{References}

[1] UK National Grid, 2014 Future Energy Scenario.

[2] UK National Grid, Electricity Ten Year Statement.

[3] UK National Grid, 2014 System Operability Framework.

[4] ENTSO-E, Network Code for Requirements for Grid Connection Applicable to all Generators.

[5] ENTSO-E, Network Code on HVDC Connections.

[6] UK National Grid, The Grid Code. Available: $\mathrm{http}: / / \mathrm{www} 2$. nationalgrid.com/UK/Industry-information/Electricitycodes/Grid-code/The-Grid-code/. [Accessed: 18-Apr-2015].

[7] UK legislation, The Electricity Supply Regulations 1988. Available:http://www.legislation.gov.uk/uksi/1988/1057/contents/made .[Accessed: 21-Jun-2015].

[8] Howard F. Illian, Frequency Control Performance Measurement and Requirements, Ernest Orlando Lawrence Berkeley National Laboratory, Dec 2010.

[9] Johnson Antony, Requirements for system inertia: simulated inertia, presented at the Grid Code Frequency Response Woking Group (UK National Grid).

[10] Ofgem, National Electricity Transmission System Security and Quality of Supply Standard (NETS SQSS): Review of Infeed Losses (GSR007 as revised by GSR007-1), Jan 2011.

[11] F. Gonzalez-Longatt, E. Chikuni, and E. Rashayi, Effects of the Synthetic Inertia from wind power on the total system inertia after a frequency disturbance, 2013 IEEE International Conference on Industrial Technology (ICIT), pp. 826-832.

[12] A. Overjordet, Synthetic inertia from wind farms - Impacts on rotor angle stability in existing synchronous generators, Master Thesis of Energy and Environmental Engineering, Norwegian University of Science and Technology (NTNU), 2014.

[13] M. Seyedi, The utilization of synthetic inertia from wind farms and its impact on existing speed governors and system performance, Jan 2013.

[14] J. Zhu, J. M. Guerrero, W. Hung, C. D. Booth, and G. P. Adam, Generic inertia emulation controller for multi-terminal voltage-source-converter high voltage direct current systems, IET Renewable Power Generation, vol. 8, no. 7, pp. 740-748, Sep. 2014.

[15] H.-P. Beck and R. Hesse, Virtual synchronous machine, 2007 9th International Conference on Electrical Power Quality and Utilisation.

[16] Q. Zhong and G. Weiss, Synchronverters: Inverters That Mimic Synchronous Generators, IEEE Transaction on Industrial Electronics, vol. 58, no. 4, pp. 1259-1267, Apr 2011.

[17] J. Driesen and K. Visscher, Virtual synchronous generators, 2008 IEEE Power and Energy Society General Meeting Conversion and Delivery of Electrical Energy in the 21st Century, pp. 1-3.

[18] H. Urdal, R. Ierna, J. Zhu, C. Ivanov, A. Dahresobh, and D. Rostom, System strength considerations in a converter 
dominated power system, IET Renewable Power Generation, vol. 9, no. 1, pp. 10-17, 2015.

[19] P. Kundur, N. J. Balu, and M. G. Lauby, Power system stability and control. New York: McGraw-Hill, 1994. 